PROCEEDINGS OF THE

AMERICAN MATHEMATICAL SOCIETY

Volume 138, Number 6, June 2010, Pages 2087-2094

S 0002-9939(10)10224-X

Article electronically published on February 5, 2010

\title{
POISSON-PINSKER FACTOR AND INFINITE MEASURE PRESERVING GROUP ACTIONS
}

\author{
EMMANUEL ROY
}

(Communicated by Bryna Kra)

\begin{abstract}
We solve the problem of the existence of a Poisson-Pinsker factor for a conservative ergodic infinite measure preserving action of a countable amenable group by proving the following dichotomy: either the system has totally positive Poisson entropy (and is of zero type) or it possesses a PoissonPinsker factor. If $G$ is abelian and the entropy positive, the spectrum is absolutely continuous (Lebesgue countable if $G=\mathbb{Z}$ ) on the whole $L^{2}$-space in the first case and in the orthocomplement of the $L^{2}$-space of the Poisson-Pinsker factor in the second.
\end{abstract}

\section{INTRODUCTION}

Poisson entropy for a $\sigma$-finite measure preserving $\mathbb{Z}$-action was introduced in [10] as the Kolmogorov entropy of its Poisson suspension (see Section 2 for the definition of a Poisson suspension); it is a non-trivial invariant which coincides with Kolmogorov entropy in the finite measure case and retains most of its basic features. The definition readily extends to more general group actions once it is well defined in the finite measure preserving case. For conservative $\mathbb{Z}$-actions, there exist other definitions of entropy due to Krengel and Parry (see [8 and 9]) and it is shown in 7 that these two entropies equal Poisson entropy for a large family of systems called "quasi-finite"; however it has been proved recently in 3 . that Krengel entropy can be different from Poisson and Parry entropies. The question of the existence of a Pinsker factor for the Krengel entropy (a Krengel-Pinsker factor) was already raised (and unsolved) in Krengel's original paper. The first partial answer was due to Aaronson and Park in [1], where they proved the existence of a Krengel-Pinsker factor for LLB systems (a subfamily of quasi-finite systems). In [7, it is proved that whenever there exists a ( $\sigma$-finite) factor of zero Poisson entropy, there exists a maximal factor with zero Poisson entropy (a Poisson-Pinsker factor).

The method used to obtain the existence of a Poisson-Pinsker factor is very specific to $\mathbb{Z}$-actions and is only partially satisfactory since it has not been possible to prove that a system generated by a square integrable function with singular

Received by the editors March 29, 2009, and, in revised form, September 22, 2009.

2010 Mathematics Subject Classification. Primary 37A40, 37A35, 60G51; Secondary 37A15, $37 \mathrm{~A} 50$

Key words and phrases. Poisson suspensions, infinite ergodic theory, joinings.

This paper was written during the MSRI semester program "Ergodic Theory and Additive Combinatorics" in Berkeley. The author is very grateful to this institution and to the organizers of this program for funding his research during this semester.

(C)2010 American Mathematical Society Reverts to public domain 28 years from publication 
spectral measure, or a conservative system with finite spectral multiplicity, has zero Poisson entropy, as is well known in the finite measure case. In this short paper, we will get, with completely different methods, a much more satisfactory picture of the situation (extended to countable amenable group actions) by showing, more generally, that the Pinsker factor of a Poisson suspension, if not trivial, is always (isomorphic to) a Poisson suspension.

These results strongly rely on the structure of joinings of Poisson suspensions (see [4, 10] and [1]) on the one hand, and entropy results for countable amenable group actions on the other (see [6], [5] and [2]), in particular the relative disjointness results of Thouvenot [12] for $\mathbb{Z}$-actions, generalized in [6] for countable amenable group actions.

\section{Background on Poisson suspensions}

We recall a few facts about Poisson suspensions that can be found in 11. Note that in this section, we mention structural features and results that had been proved in the case of $\mathbb{Z}$-actions; however, it can be seen that the extension to more general actions is immediate. In the following $G$ is a countable amenable group with identity element $e$.

2.1. Definition of Poisson suspensions. Let $(X, \mathcal{A}, \mu)$ be a $\sigma$-finite Lebesgue space with an infinite continuous measure $\mu$. Let us define the probability space $\left(X^{*}, \mathcal{A}^{*}, \mu^{*}\right)$ where:

- $X^{*}$ stands for the space of measures of the form $\nu=\sum_{n \in \mathbb{N}} \delta_{x_{i}}$ where $x_{i} \in X$.

- $\mathcal{A}^{*}$ is the smallest $\sigma$-algebra such that, for any $A \in \mathcal{A}$, the map $N_{A}: \nu \mapsto$ $\nu(A)$ from $(X, \mathcal{A})$ to $(\overline{\mathbb{N}}, \mathcal{P}(\overline{\mathbb{N}}))$ is measurable.

- $\mu^{*}$ is the only probability measure on $\left(X^{*}, \mathcal{A}^{*}\right)$ such that, for any integer $k$ and any collection of disjoint sets $\left\{A_{1}, \ldots, A_{k}\right\}$ of finite measure, the random variables $N_{A_{1}}, \ldots, N_{A_{k}}$ are independent and follow a Poisson distribution with parameters $\mu\left(A_{1}\right), \ldots, \mu\left(A_{k}\right)$ respectively.

From now on, $\mathcal{A}^{*}$ is assumed to be complete with respect to $\mu^{*}$; the probability space $\left(X^{*}, \mathcal{A}^{*}, \mu^{*}\right)$ is a Lebesgue space called the Poisson measure over $(X, \mathcal{A}, \mu)$.

Let $G$ be a countable group acting on $(X, \mathcal{A}, \mu)$ by measure preserving automorphisms $T^{g}, g \in G$. If $T$ is a measure preserving automorphism of $(X, \mathcal{A}, \mu)$, then $T_{*}$ defined on $\left(X^{*}, \mathcal{A}^{*}\right)$ by $T_{*}(\nu)(A)=\nu\left(T^{-1} A\right), A \in \mathcal{A}$, is a measure preserving automorphism of $\left(X^{*}, \mathcal{A}^{*}, \mu^{*}\right)$. $\left(X^{*}, \mathcal{A}^{*}, \mu^{*}, T_{*}^{g}\right)$ is called the Poisson suspension over the base $\left(X, \mathcal{A}, \mu, T^{g}\right)$.

2.2. Poisson entropy. The Poisson entropy of a system $\left(X, \mathcal{A}, \mu, T^{g}\right)$ is defined as the usual (Kolmogorov) entropy of its Poisson suspension $\left(X^{*}, \mathcal{A}^{*}, \mu^{*}, T_{*}^{g}\right)$. The base $\left(X, \mathcal{A}, \mu, T^{g}\right)$ is said to have totally positive Poisson entropy if for any invariant set $K \subset X$ of positive measure the Poisson entropy of the restricted system $\left(K, \mathcal{A}_{\mid K}, \mu_{\mid K}, T^{g}\right)$ is positive on ANY factor. Note that we reserve the term factor for invariant sub- $\sigma$-algebras on which the measure is still $\sigma$-finite (the trivial $\sigma$-algebra $\{X, \emptyset\} \subset \mathcal{A}$ is NOT a factor of $\left.\left(X, \mathcal{A}, \mu, T^{g}\right)\right)$; therefore, complete positive entropy (cpe) is a different notion which is not adapted to the infinite measure context. Of course a probability measure preserving system never has totally positive entropy since the trivial algebra is a factor. 
2.3. Fock space structure. It is conventional to see $L^{2}\left(\mathcal{A}^{*}\right)$ as the Fock space over $L^{2}(\mathcal{A})$, that is:

$$
L^{2}\left(\mathcal{A}^{*}\right) \simeq \mathbb{C} \oplus L^{2}(\mathcal{A}) \oplus L^{2}(\mathcal{A})^{\odot 2} \otimes \cdots \otimes L^{2}(\mathcal{A})^{\odot n} \otimes \cdots
$$

where $L^{2}(\mathcal{A})^{\odot n}$ stands for the symmetric tensor product of $L^{2}(\mathcal{A})$ and is called the $n$-th chaos. Within $L^{2}\left(\mathcal{A}^{*}\right)$, the subspace corresponding to the first chaos is denoted by $\mathfrak{C}$ and is the closure of the linear span of vectors $N(A)-\mu(A)$, for $A \in \mathcal{A}$ with $\mu(A)<\infty$. A linear operator $U$ on $L^{2}(\mathcal{A})$ of norm less than 1 induces an operator $\widetilde{U}$ on $L^{2}\left(\mathcal{A}^{*}\right)$ when defined on $L^{2}(\mathcal{A})^{\odot n}$ by $\widetilde{U}(f \otimes \cdots \otimes f)=U f \otimes \cdots \otimes U f$. We have in particular $\widetilde{U_{T^{g}}}=U_{T_{*}^{g}}$.

There is a distinguished family of vectors that is linearly dense in $L^{2}\left(\mathcal{A}^{*}\right)$, namely, for $f \in L^{2}(\mathcal{A})$ which are finite linear combinations of indicator functions,

$$
\mathcal{E}_{f}:=1 \oplus f \oplus \frac{1}{\sqrt{2 !}} f \otimes f \oplus \cdots \oplus \frac{1}{\sqrt{n !}} f \otimes \cdots \otimes f,
$$

which corresponds, through the above identification, to

$$
\mathcal{E}_{f}(\nu)=\exp \left(-\int_{X} f d \mu\right) \prod_{x, \nu(x)=1}(1+f(x)), \quad \nu \in X^{*} .
$$

In the case where $G$ is abelian, if $\sigma$ is the maximal spectral type of $U_{T^{g}}$ on $L^{2}(\mathcal{A})$, then the reduced maximal spectral type of $U_{T_{*}^{g}}$ is $\sum_{k=1}^{\infty} \frac{1}{k !} \sigma^{* k}$ since the maximal spectral type on the $k$-th chaos is $\sigma^{* k}$.

2.4. Poissonian factors. A Poissonian factor is a sub- $\sigma$-algebra of $\mathcal{A}^{*}$ of the form $\left(\mathcal{B}_{\mid K}\right)^{*}:=\sigma\left\{N_{A}, A \in \mathcal{B}_{\mid K}\right\}$ where $K \subset X$ is a $T^{g}$-invariant measurable set of positive $\mu$-measure and $\mathcal{B}_{\mid K}$ is a factor of the restricted system $\left(K, \mathcal{A}_{\mid K}, \mu_{\mid K}, T^{g}\right)$. In terms of systems, the factor $\left(\mathcal{B}_{\mid K}\right)^{*}$ corresponds to the Poisson suspension $\left(K^{*},\left(\mathcal{B}_{\mid K}\right)^{*},\left(\mu_{\mid K}\right)^{*}, T_{*}^{g}\right)$. The trivial factor $\left\{X^{*}, \emptyset\right\} \subset \mathcal{A}^{*}$ is also considered to be Poissonian.

2.5. Infinite divisibility and Poissonian joinings. Addition is well defined on $\left(X^{*}, \mathcal{A}^{*}\right)$ as the usual sum of measures and so is convolution of probability measures on $\left(X^{*}, \mathcal{A}^{*}\right)$. A probability measure $p$ on $\left(X^{*}, \mathcal{A}^{*}\right)$ such that, for any integer $k$, there exists a probability measure $p_{k}$ satisfying $p=p_{k} * \cdots * p_{k}$ ( $k$ terms) is said to be infinitely divisible. It is well known that $\mu^{*}$ is infinitely divisible as $\mu^{*}=$ $\left(\frac{1}{k} \mu\right)^{*} * \cdots *\left(\frac{1}{k} \mu\right)^{*}$.

Addition on the product space $\left(X^{*} \times X^{*}, \mathcal{A}^{*} \otimes \mathcal{A}^{*}\right)$ is defined coordinate-wise and so is convolution and infinite divisibility. A self-joining of a Poisson suspension $\left(X^{*}, \mathcal{A}^{*}, \mu^{*}, T_{*}^{g}\right)$ is said to be a Poissonian self-joining if its distribution on $\left(X^{*} \times X^{*}, \mathcal{A}^{*} \otimes \mathcal{A}^{*}\right)$ is infinitely divisible. In [1], the following is proved:

Proposition 1. A self-joining determined by a Markov operator $\Psi$ on $L^{2}\left(\mathcal{A}^{*}\right)$ is a Poissonian self-joining if and only if there exists a sub-Markov operator $\Phi$ on $L^{2}(\mathcal{A})$ (i.e. a positive operator such that $\Phi(1) \leq 1$ and $\Phi^{*}(1) \leq 1$ ) such that $\widetilde{\Phi}=\Psi$ (see [4 and 11]).

Poissonian self-joinings were originally introduced independently through their Markov operator characterization in [4] and through their infinitely divisible one in [10. 
The next proposition is found in [11] however, we give a proof, as the original one is too sketchy:

Proposition 2. If $\mathcal{F}$ is a non-trivial factor of $\left(X^{*}, \mathcal{A}^{*}, \mu^{*}, T_{*}^{g}\right)$ and if the relatively independent product of $\left(X^{*}, \mathcal{A}^{*}, \mu^{*}, T_{*}^{g}\right)$ over $\mathcal{F}$ is a Poissonian joining, then $\mathcal{F}$ is a Poissonian factor; that is, there exists a $T^{g}$-invariant subset $K \subset X$ and a factor $\mathcal{B}_{\mid K} \subset \mathcal{A}_{\mid K}$ such that $\left(\mathcal{B}_{\mid K}\right)^{*}=\mathcal{F}$.

Proof. Let $\pi_{\mathcal{F}}$ the projection on $L^{2}(\mathcal{F})$ that corresponds to the relatively independent joining over $\mathcal{F}$. Since this joining is Poissonian, there exists a sub-Markov operator $\Phi$ on $L^{2}(\mathcal{A})$ such that $\widetilde{\Phi}=\pi_{\mathcal{F}}$. Observe that $\Phi$ is also an orthogonal projection since it is induced by $\pi_{\mathcal{F}}$ restricted to the first chaos; we therefore have

$$
\int_{X} \Phi\left(1_{A}\right) d \mu=\int_{X} 1_{A} \Phi(1) d \mu .
$$

But we also have

and this implies that

$$
\int_{X} \Phi\left(1_{A}\right) d \mu=\int_{X} \Phi\left(\Phi\left(1_{A}\right)\right) d \mu=\int_{X} \Phi\left(1_{A}\right) \Phi(1) d \mu,
$$

$$
\int_{X} \Phi\left(1_{A}\right)(1-\Phi(1)) d \mu=0 .
$$

Therefore, as $\Phi$ is positive and $\Phi(1) \leq 1, \mu$-a.e. we have

$$
\Phi\left(1_{A}\right)(1-\Phi(1))=0 .
$$

Replacing $A$ by a sequence $A_{n}$ of finite measure sets increasing to $X$, we get

$$
\Phi(1)(1-\Phi(1))=0
$$

and

$$
\Phi(1)=(\Phi(1))^{2} .
$$

So $\Phi(1)$ is the indicator function of a set $K$; and if we consider the restricted system $\left(K, \mathcal{A}_{\mid K}, \mu_{\mid K}, T^{g}\right), \Phi$ is a Markov operator and an orthogonal projection of $L^{2}\left(\mathcal{A}_{\mid K}\right)$, thus a conditional expectation on a factor $\mathcal{B}_{\mid K} \subset \mathcal{A}_{\mid K}$. Now if $f \in L^{2}(\mathcal{A})$, $\mathcal{E}_{\Phi f}$ is $\left(\mathcal{B}_{\mid K}\right)^{*}$-measurable and, for any $g \in L^{2}\left(\mathcal{B}_{\mid K}\right)$,

$$
\begin{gathered}
\left\langle\pi_{\mathcal{F}} \mathcal{E}_{f}, \mathcal{E}_{g}\right\rangle_{L^{2}\left(\mathcal{A}^{*}\right)}=\left\langle\mathcal{E}_{\Phi f}, \mathcal{E}_{g}\right\rangle_{L^{2}\left(\mathcal{A}^{*}\right)}=\exp \langle\Phi f, g\rangle_{L^{2}\left(\mathcal{B}_{\mid K}\right)} \\
=\exp \langle f, g\rangle_{L^{2}\left(\mathcal{B}_{\mid K}\right)}=\exp \langle f, g\rangle_{L^{2}(\mathcal{A})}=\left\langle\mathcal{E}_{f}, \mathcal{E}_{g}\right\rangle_{L^{2}\left(\mathcal{A}^{*}\right)}=\left\langle\pi_{\mathcal{B}_{\mid K}^{*}} \mathcal{E}_{f}, \mathcal{E}_{g}\right\rangle_{L^{2}\left(\mathcal{A}^{*}\right)}
\end{gathered}
$$

with some slight abuses of notation. Therefore $\mathcal{F}=\left(\mathcal{B}_{\mid K}\right)^{*}$.

\section{The MAIN RESUlts}

Proposition 3. Let $\left(X^{*}, \mathcal{A}^{*}, \mu^{*}, T_{*}^{g}\right)$ be the Poisson suspension of the infinite measure preserving system $(X, \mathcal{A}, \mu, G)$. Then $\Pi$, the Pinsker factor of the system, is a Poissonian factor.

Proof. Take $\alpha, \beta>0$ and consider the direct product

$$
\left(X^{*} \times X^{*}, \mathcal{A}^{*} \otimes \mathcal{A}^{*},(\alpha \mu)^{*} \otimes(\beta \mu)^{*}, T_{*}^{g} \times T_{*}^{g}\right)
$$

of Poisson suspensions. Thanks to the classical formula $(\alpha \mu)^{*} *(\beta \mu)^{*}=(\alpha+\beta) \mu^{*}$, the map $\varphi:\left(\nu_{1}, \nu_{2}\right) \mapsto \nu_{1}+\nu_{2}$ induces a factor map from

$$
\left(X^{*} \times, X^{*} \mathcal{A}^{*} \otimes \mathcal{A}^{*},(\alpha \mu)^{*} \otimes(\beta \mu)^{*}, T_{*}^{g} \times T_{*}^{g}\right)
$$


to

$$
\left(X^{*}, \mathcal{A}^{*},((\alpha+\beta) \mu)^{*}, T_{*}^{g}\right) .
$$

Let $\Pi_{\alpha}, \Pi_{\beta}$ and $\Pi_{\alpha+\beta}$ be the Pinsker algebras of the systems $\left(X^{*}, \mathcal{A}^{*},(\alpha \mu)^{*}, T_{*}^{g}\right)$, $\left(X^{*}, \mathcal{A}^{*},(\beta \mu)^{*}, T_{*}^{g}\right)$ and $\left(X^{*}, \mathcal{A}^{*},((\alpha+\beta) \mu)^{*}, T_{*}^{g}\right)$ respectively and set $\mathcal{B}:=\varphi^{-1} \mathcal{A}^{*}$ and $\widetilde{\Pi_{\alpha+\beta}}:=\varphi^{-1} \Pi_{\alpha+\beta}$. Thanks to a classical result (generalized to countable amenable group actions in Theorem 4 in 6 ), the Pinsker algebra of the product $\left(X^{*} \times X^{*}, \mathcal{A}^{*} \otimes \mathcal{A}^{*},(\alpha \mu)^{*} \otimes(\beta \mu)^{*}, T_{*}^{g} \times T_{*}^{g}\right)$ is $\Pi_{\alpha} \otimes \Pi_{\beta}$. The extension $\mathcal{B} \rightarrow \widetilde{\Pi_{\alpha+\beta}}$ is a cpe extension and $\Pi_{\alpha} \otimes \Pi_{\beta} \rightarrow \widetilde{\Pi_{\alpha+\beta}}$ is a zero entropy extension. Therefore, by Lemma 3 in 12] (once again generalized to countable amenable group actions in Theorem 1 in [6]), they are relatively disjoint over $\widetilde{\Pi_{\alpha+\beta}}$. As a consequence, $L^{2}(\mathcal{B}) \ominus L^{2}\left(\widetilde{\Pi_{\alpha+\beta}}\right)$ and $L^{2}\left(\Pi_{\alpha} \otimes \Pi_{\beta}\right) \ominus L^{2}\left(\widetilde{\Pi_{\alpha+\beta}}\right)$ are orthogonal in $L^{2}\left(\mathcal{A}^{*} \otimes \mathcal{A}^{*}\right)$. Indeed if $f \in L^{2}(\mathcal{B}) \ominus L^{2}\left(\widetilde{\Pi_{\alpha+\beta}}\right)$ and $g \in L^{2}\left(\Pi_{\alpha} \otimes \Pi_{\beta}\right) \ominus L^{2}\left(\widetilde{\Pi_{\alpha+\beta}}\right)$, we have

$$
\begin{aligned}
& \mathbb{E}[f g] \\
& =\mathbb{E}\left[\mathbb{E}\left[f g \mid \widetilde{\Pi_{\alpha+\beta}}\right]\right] \\
& =\mathbb{E}\left[\mathbb{E}\left[f \mid \widetilde{\Pi_{\alpha+\beta}}\right] \mathbb{E}\left[g \mid \widetilde{\Pi_{\alpha+\beta}}\right]\right] \\
& =0 .
\end{aligned}
$$

We can therefore decompose $L^{2}\left(\mathcal{A}^{*} \otimes \mathcal{A}^{*}\right)$ into the following orthogonal sum:

$$
\begin{aligned}
& L^{2}\left(\mathcal{A}^{*} \otimes \mathcal{A}^{*}\right) \\
& =L^{2}\left(\widetilde{\Pi_{\alpha+\beta}}\right) \oplus\left(L^{2}(\mathcal{B}) \ominus L^{2}\left(\widetilde{\Pi_{\alpha+\beta}}\right)\right) \oplus\left(L^{2}\left(\Pi_{\alpha} \otimes \Pi_{\beta}\right) \ominus L^{2}\left(\widetilde{\Pi_{\alpha+\beta}}\right)\right) \oplus H
\end{aligned}
$$

where $H$ is the orthocomplement of everything else. Now write $f \in L^{2}\left(\mathcal{A}^{*} \otimes \mathcal{A}^{*}\right)$ as $f=f_{1}+f_{2}+f_{3}+f_{4}$ according to the decomposition; we have

$$
\mathbb{E}\left[\mathbb{E}[f \mid \mathcal{B}] \mid \Pi_{\alpha} \otimes \Pi_{\beta}\right]=\mathbb{E}\left[\left(f_{1}+f_{2}\right) \mid \Pi_{\alpha} \otimes \Pi_{\beta}\right]=f_{1}
$$

and thus $\mathbb{E}\left[f \mid \widetilde{\Pi_{\alpha+\beta}}\right]=\mathbb{E}\left[\mathbb{E}[f \mid \mathcal{B}] \mid \Pi_{\alpha} \otimes \Pi_{\beta}\right]$.

Now form the relatively independent joining of

$$
\left(X^{*} \times, X^{*} \mathcal{A}^{*} \otimes \mathcal{A}^{*},(\alpha \mu)^{*} \otimes(\beta \mu)^{*}, T_{*}^{g} \times T_{*}^{g}\right)
$$

over $\Pi_{\alpha} \otimes \Pi_{\beta}$ and observe that it is just the direct product of the relatively independent joinings of $\left(X^{*}, \mathcal{A}^{*},(\alpha \mu)^{*}, T_{*}^{g}\right)$ and $\left(X^{*}, \mathcal{A}^{*},(\beta \mu)^{*}, T_{*}^{g}\right)$ over their respective Pinsker factors $\Pi_{\alpha}$ and $\Pi_{\beta}$. Let's now compute the distribution of the self-joining of $\left(X^{*}, \mathcal{A}^{*}, \mu^{*}, T_{*}^{g}\right)$ induced by the preceding joining through the factor map $\varphi \times \varphi$. Take $A$ and $B$ in $\mathcal{B}$ and compute:

$$
\begin{aligned}
& \mathbb{E}\left[\mathbb{E}\left[1_{A} \mid \Pi_{\alpha} \otimes \Pi_{\beta}\right] \mathbb{E}\left[1_{B} \mid \Pi_{\alpha} \otimes \Pi_{\beta}\right]\right] \\
& =\mathbb{E}\left[\mathbb{E}\left[\mathbb{E}\left[1_{A} \mid \mathcal{B}\right] \mid \Pi_{\alpha} \otimes \Pi_{\beta}\right] \mathbb{E}\left[\mathbb{E}\left[1_{B} \mid \mathcal{B}\right] \mid \Pi_{\alpha} \otimes \Pi_{\beta}\right]\right] \\
& =\mathbb{E}\left[\mathbb{E}\left[1_{A} \mid \widetilde{\Pi_{\alpha+\beta}}\right] \mathbb{E}\left[1_{B} \mid \widetilde{\Pi_{\alpha+\beta}}\right]\right] .
\end{aligned}
$$

Therefore, the joining induced is none other than the relatively independent joining over its Pinsker factor. We have just proved that the image measure of

$$
\left((\alpha \mu)^{*} \otimes_{\Pi_{\alpha}}(\alpha \mu)^{*}\right) \otimes\left((\beta \mu)^{*} \otimes_{\Pi_{\beta}}(\beta \mu)^{*}\right)
$$


by the sum application $\varphi \times \varphi$ is the measure $\mu^{*} \otimes_{\Pi_{\alpha+\beta}} \mu^{*}$; that is, we have proved the formula

$$
\left((\alpha \mu)^{*} \otimes_{\Pi_{\alpha}}(\alpha \mu)^{*}\right) *\left((\beta \mu)^{*} \otimes_{\Pi_{\beta}}(\beta \mu)^{*}\right)=\mu^{*} \otimes_{\Pi_{\alpha+\beta}} \mu^{*}
$$

and we can deduce that, for any integer $k$,

$$
\left(\left(\frac{1}{k} \mu\right)^{*} \otimes_{\Pi_{\frac{1}{k}}}\left(\frac{1}{k} \mu\right)^{*}\right)^{* k}=\mu^{*} \otimes_{\Pi_{1}} \mu^{*} .
$$

This means that the distribution of this relatively independent joining is infinitely divisible; i.e. it is a Poissonian joining. But according to Proposition 2, $\Pi_{1}=\Pi$ is a Poissonian factor.

Proposition 4. Let $\left(X, \mathcal{A}, \mu, T^{g}\right)$ be a dynamical system. There exists a (possibly trivial) partition into $T^{g}$-invariant sets $A$ and $A^{c}$ such that:

(1) $\left(A, \mathcal{A}_{\mid A}, \mu_{\mid A}, T^{g}\right)$ possesses a Poisson-Pinsker factor;

(2) for any $T^{g}$-invariant set $B \subset A^{c}$ of positive measure, $\left(B, \mathcal{A}_{\mid B}, \mu_{\mid B}, T^{g}\right)$ has totally positive Poisson entropy.

Proof. If the Pinsker factor of $\left(X^{*}, \mathcal{A}^{*}, \mu^{*}, T_{*}^{g}\right)$ is trivial, the system has complete positive entropy, and therefore, for any $T^{g}$-invariant set $B \subset X$ of positive measure, $\left(B, \mathcal{A}_{\mid B}, \mu_{\mid B}, T^{g}\right)$ has totally positive Poisson entropy since any factor $\mathcal{B}_{\mid B}$ corresponds to the non-trivial Poissonian factor $\left(\mathcal{B}_{\mid B}\right)^{*}$ on which the Poisson suspension has positive entropy. If the Pinsker factor $\Pi$ of $\left(X^{*}, \mathcal{A}^{*}, \mu^{*}, T_{*}^{g}\right)$ is not trivial, by Proposition 3 there exists a $T^{g}$-invariant set $A \subset X$ of positive measure and a factor, say $\mathcal{P}_{\mid A}$, of the restricted system $\left(A, \mathcal{A}_{\mid A}, \mu_{\mid A}, T^{g}\right)$ such that $\left(\mathcal{P}_{\mid A}\right)^{*}=\Pi$. $\mathcal{P}_{\mid A}$ is clearly the Poisson-Pinsker factor of the system $\left(A, \mathcal{A}_{\mid A}, \mu_{\mid A}, T^{g}\right)$. Indeed, if $\mathcal{C}_{\mid A} \subset \mathcal{A}_{\mid A}$ is a factor with zero Poisson entropy, then $\left(\mathcal{C}_{\mid A}\right)^{*} \subset\left(\mathcal{P}_{\mid A}\right)^{*}$ as the latter is the Pinsker factor of the suspension, and this implies that $\mathcal{C}_{\mid A} \subset \mathcal{P}_{\mid A}$ (the fact that a factor $\mathcal{R}$ is the Poisson-Pinsker factor if the associated Poissonian factor $\mathcal{R}^{*}$ is the Pinsker factor of the suspension was already observed in [7]).

If $A^{c}$ has positive measure, the Poisson suspension $\left(X^{*}, \mathcal{A}^{*}, \mu^{*}, T_{*}^{g}\right)$ splits into the direct product

$$
\left(A^{*} \times\left(A^{c}\right)^{*},\left(\mathcal{A}_{\mid A}\right)^{*} \otimes\left(\mathcal{A}_{\mid A^{c}}\right)^{*},\left(\mu_{\mid A}\right)^{*} \otimes\left(\mu_{\mid A^{c}}\right)^{*}, T_{*}^{g} \times T_{*}^{g}\right),
$$

which implies that the Pinsker factor $\Pi$ also splits accordingly. But as $\left(\mathcal{P}_{\mid A}\right)^{*}=$ $\Pi$, the Pinsker factor is concentrated in the left half of the product; that is, $\left(\left(A^{c}\right)^{*},\left(\mathcal{A}_{\mid A^{c}}\right)^{*},\left(\mu_{\mid A^{c}}\right)^{*}, T_{*}^{g}\right)$ has complete positive entropy, and we conclude as in the first part of the proof.

In the ergodic case, the result takes the following more pleasant form:

Theorem 5. Let $\left(X, \mathcal{A}, \mu, T^{g}\right)$ be an ergodic infinite measure preserving system. Either it has totally positive Poisson entropy, or it possesses a Poisson-Pinsker factor.

As in the $\mathbb{Z}$-action case, we can observe the behaviour of Poisson entropy with respect to joinings.

Proposition 6. Zero Poisson entropy is stable under taking joinings. Totally positive entropy systems are strongly disjoint from systems possessing a PoissonPinsker factor. 
Proof. The first statement is obvious, and the proof of the second is identical to that in the $\mathbb{Z}$-action case, which can be found in [7].

\section{Spectral properties}

We first recall that a system $\left(X, \mathcal{A}, \mu, T^{g}\right)$ is of zero type if for any measurable sets $A$ and $B$ in $\mathcal{A}$ of finite measure, $\mu\left(A \cap T^{g} B\right)$ tends to zero as $g$ tends to infinity.

Proposition 7. If $\left(X, \mathcal{A}, \mu, T^{g}\right)$ has totally positive entropy, then it is of zero type.

Proof. From Proposition $3\left(X^{*}, \mathcal{A}^{*}, \mu^{*}, T_{*}^{g}\right)$ has complete positive entropy and is therefore mixing. But, thanks to the classical isometry formula

$$
\mu(A \cap B)=\mathbb{E}_{\mu^{*}}\left[\left(N_{A}-\mu(A)\right)\left(N_{B}-\mu(B)\right)\right],
$$

we have

$$
\begin{aligned}
& \mu\left(A \cap T^{g} B\right) \\
& =\mathbb{E}_{\mu^{*}}\left[\left(N_{A}-\mu(A)\right)\left(N_{T^{g} B}-\mu\left(T^{g} B\right)\right)\right] \\
& =\mathbb{E}_{\mu^{*}}\left[\left(N_{A}-\mu(A)\right)\left(N_{B}-\mu(B)\right) \circ T_{*}^{g}\right],
\end{aligned}
$$

which goes to zero as $g$ tends to infinity.

Proposition 8. If $G$ is abelian and $\left(X^{*}, \mathcal{A}^{*}, \mu^{*}, T_{*}^{g}\right)$ has positive entropy, then it has absolutely continuous spectrum on $\mathfrak{C} \cap\left(L^{2}(\Pi)^{\perp}\right)$.

Proof. Since, thanks to Proposition 3, $\Pi$ has the structure of a Poissonian factor, its associated $L^{2}$-space is a Fock space compatible with the underlying one; in particular $\mathfrak{C}=\left(L^{2}(\Pi)^{\perp} \cap \mathfrak{C}\right) \stackrel{\perp}{\oplus}\left(L^{2}(\Pi) \cap \mathfrak{C}\right)$. Since by assumption $L^{2}(\Pi) \neq$ $L^{2}\left(\mathcal{A}^{*}\right), \mathfrak{C} \not \subset L^{2}(\Pi)$ as $\sigma(\mathfrak{C})=\mathcal{A}^{*}$. Therefore $\left(L^{2}(\Pi)^{\perp} \cap \mathfrak{C}\right)$ is not empty and we get the result, as the maximal spectral type on $L^{2}(\Pi)^{\perp}$ is Lebesgue by a theorem proved in [5] and independently by Thouvenot (unpublished).

As a direct application, we get:

Proposition 9. Assume $G$ is abelian. If $\left(X, \mathcal{A}, \mu, T^{g}\right)$ has totally positive Poisson entropy, its maximal spectral type is absolutely continuous.

If $\left(X, \mathcal{A}, \mu, T^{g}\right)$ has positive Poisson entropy and possesses a Poisson-Pinsker factor, then the maximal spectral type on the orthocomplement of the PoissonPinsker factor is absolutely continuous.

Proof. This follows directly from Proposition 8 and from the unitary isomorphism between the first chaos of $L^{2}\left(\mathcal{A}^{*}\right)$ and $L^{2}(\mathcal{A})$.

We can therefore deduce the following corollary, which is well known in the finite measure case, with the proof being almost the same:

Corollary 10. Assume $\left(X, \mathcal{A}, \mu, T^{g}\right)$ is the dynamical system associated to a square integrable stationary $G$-process $\left\{X_{g}\right\}_{g \in G}$ where $G$ is a countable abelian group. If the spectral measure of $X_{e}$ is singular, then $\left(X, \mathcal{A}, \mu, T^{g}\right)$ has zero Poisson entropy.

In particular, if $\left(X, \mathcal{A}, \mu, T^{g}\right)$ has singular maximal spectral type, then it has zero Poisson entropy.

For a $\mathbb{Z}$-action, we can be more precise. 
Proposition 11. If $(X, \mathcal{A}, \mu, T)$ has totally positive Poisson entropy, its maximal spectral type is Lebesgue countable.

If $(X, \mathcal{A}, \mu, T)$ has positive Poisson entropy and possesses a Poisson-Pinsker factor, then the maximal spectral type on the orthocomplement of the Poisson-Pinsker factor is Lebesgue countable.

Proof. The first statement follows from Proposition 10.2 in [7, combined with Proposition 9. The second can also be deduced from an adaptation of Proposition 10.2 in [7] but is also a direct application of Theorem 3.2 in [2] combined with the fact that conditional Poisson entropy coincides with conditional Krengel entropy as proved in [7].

Examples of totally positive Poisson entropy transformations are given by shifts associated to null recurrent Markov chains (see [11).

Corollary 12. If $(X, \mathcal{A}, \mu, T)$ has finite multiplicity, then it has zero Poisson entropy.

The conclusion of Corollary 12 is not true if $\mu$ is not continuous (think of the shift on $\mathbb{Z})$.

\section{REFERENCES}

1. J. Aaronson and K. K. Park, Predictability, entropy and information of infinite transformations, Fund. Math. 206 (2009), 1-21.

2. I. A. Danilenko and D. J. Rudolph, Conditional entropy theory in infinite measure and a question of Krengel, Israel J. Math. 172 (2009), no. 1, 93-117.

3. T. de la Rue and E. Janvresse, Krengel entropy does not kill Poisson entropy, preprint.

4. Y. Derriennic, K. Frączek, M. Lemańczyk, and F. Parreau, Ergodic automorphisms whose weak closure of off-diagonal measures consists of ergodic self-joinings, Colloq. Math. 110 (2008), 81-115. MR.2353900 (2008j:37001)

5. A. H. Dooley and V. Ya. Golodets, The spectrum of completely positive entropy actions of countable amenable groups, J. Funct. Anal. 196 (2002), 1-18. MR1941988 (2003m:37006)

6. E. Glasner, J.-P. Thouvenot, and B. Weiss, Entropy theory without a past, Ergodic Theory Dynam. Systems 20 (2000), 1355-1370. MR1786718 (2001h:37011)

7. E. Janvresse, T. Meyerovitch, T. de la Rue, and E. Roy, Poisson suspensions and entropy for infinite transformations, Trans. Amer. Math. Soc., to appear.

8. U. Krengel, Entropy of conservative transformations, Z. Wahrscheinlichkeitstheorie und Verw. Geb. 7 (1967), 161-181. MR0218522 (36:1608)

9. W. Parry, Entropy and generators in ergodic theory, W. A. Benjamin, Inc., New YorkAmsterdam, 1969. MR0262464(41:7071)

10. E. Roy, Mesures de Poisson, infinie divisibilité et propriétés ergodiques, Ph.D. thesis, Université Paris 6, 2005.

11. 29 (2009), no. 2, 667-683. MR.2486789

12. J.-P. Thouvenot, Une classe de systèmes pour lesquels la conjecture de Pinsker est vraie, Israel J. Math. 21 (1975), no. 2-3, 208-214. MR0382602 (52:3484)

Laboratoire Analyse Géométrie et Applications, UMr 7539, Université Paris 13, 99 avenue J.B. Clément, F-93430 Villetaneuse, France

E-mail address: roy@math.univ-paris13.fr 